\title{
Hepatoprotective activity of chrysin is mediated through TNF- $\alpha$ in chemically-induced acute liver damage: An in vivo study and molecular modeling
}

\author{
ANCA HERMENEAN ${ }^{1,2}$, TEODORA MARIASIU ${ }^{1}$, INMACULADA NAVARRO-GONZÁLEZ ${ }^{3}$, \\ JOSEFINA VEGARA-MESEGUER ${ }^{4}$, EFTIMIE MIUȚESCU ${ }^{5}$, \\ SANDIPAN CHAKRABORTY ${ }^{6}$ and HORACIO PÉREZ-SÁNCHEZ ${ }^{4}$
}

\begin{abstract}
${ }^{1}$ Department of Histology, Faculty of Medicine and ${ }^{2}$ Institute of Life Sciences, Vasile Goldis Western University of Arad, 310414 Arad, Romania; ${ }^{3}$ Department of Food Science and Nutrition, Faculty of Veterinary Science,

Campus de Espinardo, University of Murcia, 30100 Murcia; ${ }^{4}$ Bioinformatics and High Performance

Computing Research Group, Saint Anthony Catholic University of Murcia, 30107 Murcia, Spain;

${ }^{5}$ Department of Gastroenterology, Faculty of Medicine, Vasile Goldis Western University of Arad, 310414 Arad,

Romania; ${ }^{6}$ Department of Microbiology, University of Calcutta, Kolkata, West Bengal 700019, India
\end{abstract}

Received May 24, 2016; Accepted August 18, 2016

DOI: $10.3892 / \mathrm{etm} .2017 .4181$

\begin{abstract}
Chrysin (5,7-dihydroxyflavone) is a naturally occurring flavonoid present at high levels in honey, propolis and numerous plant extracts. Chrysin is known to have hepatoprotective activity, however, the mechanisms by which it exerts this effect remain unclear. In the present study, the effects of chrysin in carbon tetrachloride $\left(\mathrm{CCl}_{4}\right)$-induced acute liver damage were investigated and the results used to infer a possible mechanism behind chrysin's hepatoprotective activity. Prior to an intraperitoneal injection of $\mathrm{CCl}_{4}(1 \mathrm{ml} / \mathrm{kg})$ to induce acute liver damage, chrysin $(50 \mathrm{mg} / \mathrm{kg})$ was administered orally to mice for 7 days. The positive control group was given $50 \mathrm{mg} / \mathrm{kg}$ standardized silymarin, a well-studied hepatoprotective flavonoid. Twenty-four $\mathrm{h}$ following $\mathrm{CCl}_{4}$ administration, an increase in the activity levels of serum aspartate-amino-transferase and alanine-amino-transferase was found. This was accompanied by extended centrilobular necrosis, steatosis and an altered hepatocyte ultrastructure. In addition, $\mathrm{CCl}_{4}$-induced acute hepatotoxicity was associated
\end{abstract}

Correspondence to: Professor Anca Hermenean, Department of Histology, Faculty of Medicine, Vasile Goldis Western University of Arad, 86 Rebreanu Street, 310414 Arad, Romania

E-mail: anca.hermenean@gmail.com

Professor Horacio Pérez-Sánchez, Bioinformatics and High Performance Computing Research Group, Saint Anthony Catholic University of Murcia, Campus de los Jerónimos, 30107 Murcia, Spain

E-mail: hperez@ucam.edu

Key words: chrysin, liver, hepatoprotection, tumor necrosis factor- $\alpha$, molecular modeling, carbon tetrachloride, $\alpha$-smooth muscle actin, silymarin with an increase in hepatic tumor necrosis factor- $\alpha$ (TNF- $\alpha$ ) and $\alpha$-smooth muscle actin ( $\alpha$-SMA) protein expression, which was significantly decreased in the livers of mice pre-treated with chrysin $(\mathrm{P}<0.001)$, similar to the results of the silymarin pre-treated group $(\mathrm{P}<0.001)$. Treatment with chrysin prior to $\mathrm{CCl}_{4}$ exposure significantly reduced the activity of enzymes used as biochemical markers of poor liver function compared with the group which did not receive pre-treatment $(\mathrm{P}<0.001)$. In addition, the results of histopathological and electron microscopy liver examination showed chrysin pre-treatment reduced the effects of $\mathrm{CCl}_{4}$ treatment. Molecular modeling results demonstrated that the hepatoprotective activity of chrysin is mediated through TNF- $\alpha$, as it reduces soluble TNF- $\alpha$ generation via blocking TNF- $\alpha$-converting enzyme activity. In conclusion, the results of the present study suggest that inflammatory pathways are activated in $\mathrm{CCl}_{4}$-induced acute liver damage, which are ameliorated by chrysin pre-treatment. This indicates that chrysin is a potent hepatoprotective agent, similarly to silymarin at the same dose, which has the potential to be a viable alternative to conventional hepatoprotective treatments.

\section{Introduction}

The liver is the primary organ responsible for the metabolism of numerous xenobiotics, including drugs and toxic chemicals (1). Carbon tetrachloride $\left(\mathrm{CCl}_{4}\right)$, an industrial solvent, cleaner and degreaser, has been used extensively in models of xenobiotic-induced hepatotoxicity (2). $\mathrm{CCl}_{4}$-induced liver damage is characterized by progressive tissue injury, starting with inflammation and followed by necrosis, fibrosis and, finally, cirrhosis $(2,3)$. Acute inflammation triggers further inflammatory processes, initiated by cytokines released from activated Kupffer cells. This represents a key event in the induction of liver damage. Previous studies have demonstrated 
that inflammation is initiated by the release of pro-inflammatory mediators, including TNF- $\alpha$, cyclooxygenase- 2 and interleukin-6, in response to oxidative stress conditions, such as those during $\mathrm{CCl}_{4}$-induced hepatotoxicity, and occurs in parallel with increasing apoptosis $(4,5)$. TNF- $\alpha$, the primary pro-inflammatory protein synthesized by Kupffer cells, initiates a cascade of cytokines that mediate the inflammatory response (6).

A previous study found an elevated expression of TNF- $\alpha$ in the early phases of liver damage (7). The next stage in $\mathrm{CCl}_{4}$-induced liver damage is activation of hepatic stellate cells (HSCs) (8). HSCs are myofibroblasts that reside in a heterogeneous cell population originating from liver fibroblasts and bone marrow-derived circulating fibroblasts. Active HSCs are characterized by a high rate of proliferation, migration and contractility (9). In addition, HSCs are the primary producers of $\alpha$-SMA, which activates the production of transforming growth factor-b1, the primary pro-fibrogenic cytokine (10). These events result in collagen deposition and the release of other matrix proteins into the extracellular space, promoting liver fibrosis.

Natural antioxidants can prevent liver damage by scavenging free radicals and other reactive oxygen species, or by modulation of the inflammatory response (11). Additionally, a previous study has shown that xenobiotic-induced hepatotoxicity is diminished by flavonoids, such as silymarin (12). Silymarin, the primary bioactive compound of Silybum marianum, is a complex mixture of flavonolignans, which has protective effects against xenobiotics, particularly in the liver (13). The hepatoprotective activity of silymarin is a result of its antioxidant properties, lipid peroxidation inhibition and cell membrane preservation (14). Chrysin (5,7-dihydroxyflavone) is another natural flavonoid. Chrysin has not been as well-studied as silymarin, but is known to be present in high levels in honey, propolis and numerous plant extracts (15). Chrysin has been identified to possess antioxidant (16-18), anti-allergic (19), anti-inflammatory (20), anti-fibrotic (21) and anti-cancer $(22,23)$ properties. However, there are previous reports in the literature regarding the hepatoprotective activity of chrysin, but these did not reveal how its protective activity is initiated in acute liver damage condition.

In the present study, the hepatoprotective effects of chrysin against acute $\mathrm{CCl}_{4}$-induced liver damage are investigated and the results used to postulate a possible mechanism by which this occurs. In addition, the interaction between chrysin and TNF- $\alpha$ was evaluated by computational molecular modeling.

\section{Materials and methods}

Chemicals and reagents. Chrysin (97\%), silymarin (98\%) and carboxymethyl cellulose were purchased from Sigma-Aldrich (Merck Millipore, Darmstadt, Germany). Anti-TNF- $\alpha$ and anti- $\alpha$-SMA, antibodies were obtained from Santa Cruz Biotechnology, Inc. (Dallas, TX, USA). The Novolink Max Polymer Detection System for immunohistochemistry was purchased from Leica Microsystems GmbH (Wetzlar, Germany).

Experimental animals. A total of 50 male CD-1 mice (weight, $20 \pm 3 \mathrm{~g}$; age, 8-10 weeks), supplied by the Animal House of the Vasile Goldis Western University of Arad (Arad, Romania) were used in the present study. The animals were maintained in an environment at a constant temperature of $20 \pm 1^{\circ} \mathrm{C}$ and $50 \pm 5 \%$ humidity, with a 12-h light/dark cycle and ad libitum access to food and water. All experimental procedures were approved by the Ethical Committee of Vasile Goldis Western University of Arad (Arad, Romania).

Treatments and experimental design. A $50 \mathrm{mg} / \mathrm{kg}$ body weight dose of chrysin was chosen, as it was previously proven to be protective against oxidative damage caused by toxicants in rodents (24). Silymarin $(50 \mathrm{mg} / \mathrm{kg})$ was used as the positive control drug. Chrysin and silymarin were dissolved in $0.5 \%$ sodium carboxymethylcellulose (Sigma-Aldrich; Merck Millipore) and given orally. $\mathrm{CCl}_{4}(1.0 \mathrm{ml} / \mathrm{kg}$, in a 1:1 ratio with $50 \%$ olive oil), injected intraperitoneally, was used to induce acute liver damage.

The 50 mice were divided into 5 groups of 10 mice. Group 1 (control) received only the vehicle daily for 7 days. Group $2\left(\mathrm{CCl}_{4}\right)$ received the vehicle daily for 7 days, followed by $\mathrm{CCl}_{4}$ the next day. Group 3 (chrysin pre-treatment group; $\mathrm{CHR}+\mathrm{CCl}_{4}$ ) received chrysin for 7 days, followed by $\mathrm{CCl}_{4}$ the next day. Group 4 (silymarin pre-treatment group; $\mathrm{Sy}+\mathrm{CCl}_{4}$ ) received silymarin for 7 days, followed by $\mathrm{CCl}_{4}$ the next day. Group 5 (CHR) group, received chrysin alone for 7 days.

Serum and liver sample collection. The mice were sacrificed with $2.5 \mathrm{ml} / \mathrm{l} / \mathrm{min}$ isoflurane on day 9 and blood collected from the venae cavae. The collected blood was placed in heparinized tubes and centrifuged at room temperature for $15 \mathrm{~min}$ at $366 \times \mathrm{x}$ in order to obtain serum samples for biochemical analysis. Liver samples (2-cm samples) were preserved in a buffered formalin solution for histology and immunohistochemistry and glutaraldehyde solution for electron microscopy processing.

Biochemical analysis of the activity of serum markers of hepatic function. The activities of serum aspartate aminotransferase (AST) and alanine aminotransferase (ALT) were evaluated by spectrophotometry using a commercially available detection kits (cat. no. 11876805216; Roche Applied Science, Penzberg, Germany) according to the manufacturer's instructions.

Histopathology. Liver sections $(5-\mu \mathrm{m})$ were deparaffinized and processed routinely for hematoxylin and eosin staining. The extent of $\mathrm{CCl}_{4}$-induced hepatic damage was then evaluated by assessing morphological changes to the liver sections. Frozen sections were cut to $8 \mu \mathrm{m}$ using an MNT cryostat (SLEE medical GmbH, Mainz, Germany), fixed in $10 \%$ buffered formaldehyde and stained with Oil Red $\mathrm{O}$ according to the manufacturer's instructions. Mounted sample slides were examined under a BX43 light microscope and images captured using an XC30 digital camera (both Olympus Corporation, Tokyo, Japan).

Immunohistochemistry. Immunohistochemistry analysis was performed on paraffin-embedded $4-\mu$ m-thick liver tissue sections. Liver sections were deparaffinized in toluene and rehydrated prior to epitope retrieval in Novocastra Epitope 
A

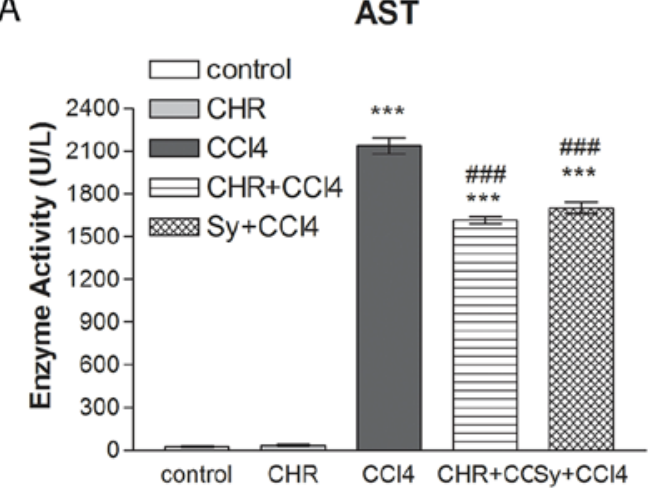

B

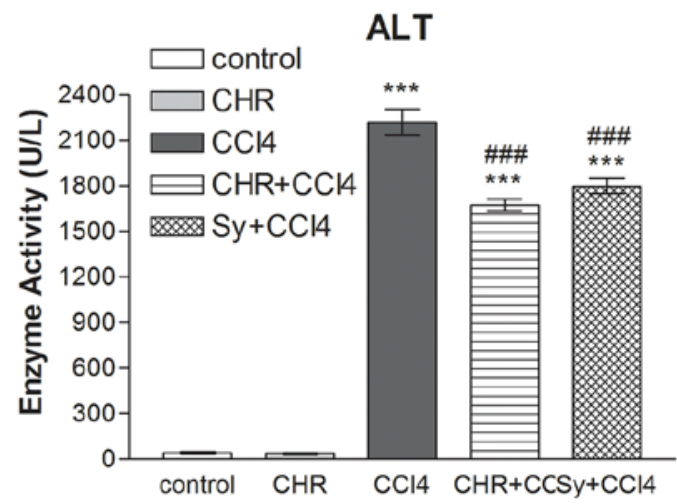

Figure 1. Effect of chrysin on the activities of serum (A) AST and (B) ALT following $\mathrm{CCl}_{4}$ treatment. Chrysin pre-treatment reduced on serum AST and ALT activities. Results are represented as the mean \pm standard deviation $(\mathrm{n}=8)$. ${ }^{* * *} \mathrm{P}<0.001$ vs. the control group; ${ }^{\# \# \#} \mathrm{P}<0.001$ vs. the $\mathrm{CCl}{ }_{4}$ group. AST, aspartate transaminase; ALT, alanine transaminase; $\mathrm{CCl}_{4}$, carbon tetrachloride; $\mathrm{CHR}$, chrysin; Sy, silymarin.

Retrieval Solution (Leica Biosystems, Nussloch, Germany). Following neutralization of endogenous peroxidase with $3 \%$ $\mathrm{H}_{2} \mathrm{O}_{2}$ for 10 min, sections were incubated with BSA blocking solution (Thermo Fisher Scientific, Inc., Waltham, MA, USA) for $30 \mathrm{~min}$ at room temperature, and then incubated at $4^{\circ} \mathrm{C}$ overnight with anti-TNF- $\alpha$ (cat. no. sc-52746; Santa Cruz Biotechnology, Inc., Dallas, TX, USA) or anti- $\alpha$-SMA (cat. no. ab32575; Abcam, Cambridge, UK) primary antibodies (1:100). Detection was then performed using a polymer detection system (cat. no. RE7280-K; Novolink Max Polymer Detection system) and 3,3'-diaminobenzidine as chromogenic substrate (both Leica Biosystems). Tissues were stained with hematoxylin, dehydrated in a gradient of alcohol and mounted onto slides. Negative control sections were processed in the same way, but with the primary antibodies substituted for immunoglobulins of the same isotype. Slides were examined and images captured as described previously. The intensity of TNF- $\alpha$ and $\alpha$-SMA immunopositivity was analyzed with ImageJ (64-bit) software v.2.1.4.6. (U.S. National Institutes of Health, Bethesda, Maryland, USA). Five fields were selected randomly from each liver section. Results are presented as the percentage of brown-stained TNF- $\alpha$ and $\alpha$-SMA positive fields compared with the control group (set to $100 \%$ ).

Electron microscopy. Liver sections for electron microscopy were prefixed in $2.7 \%$ glutaraldehyde solution in $0.1 \mathrm{M}$ phosphate buffer at $4^{\circ} \mathrm{C}$ for $1.5 \mathrm{~h}$. Then, specimens were washed in $0.15 \mathrm{M}$ phosphate buffer ( $\mathrm{pH} 7.2$ ) and fixed in $2 \%$ osmium tetroxide in $0.15 \mathrm{M}$ phosphate buffer at $4^{\circ} \mathrm{C}$ for $1 \mathrm{~h}$. Dehydration was performed in acetone and samples embedded in epoxy resin Epon 812. Embedded samples were then cut into 70-nm-thick sections with the Leica EM UC7 ultramicrotome (Leica Microsystems GmbH, Wetzlar, Germany), treated with uranyl acetate and lead citrate solutions for double contrast and analyzed with a Tecnai transmission electron microscope (FEI; Hillsboro, OR, USA).

Molecular modeling of chrysin-TNF- $\alpha$-converting enzyme (TACE or ADAM17) binding. To understand the protective effects of chrysin on $\mathrm{CCl}_{4}$-induced TNF- $\alpha$ expression, the present study performed molecular modeling to study the interaction between chrysin and TACE, the proteinase responsible for cleaving pro-TNF- $\alpha$ to generate TNF- $\alpha$. The crystal structure of the catalytic domain of TACE with a bound inhibitor (IK682) is solved and available in Protein Data Bank (PDB; PDB ID: 2FV5) (25). In the present study, the inhibitor was considered a reference ligand and the active site of the enzyme was defined as the residues within $10 \AA$ of the reference ligand.

The 3D structure of chrysin was built using the molecular builder interface of HYPERCHEM (version 8.0) (26) and optimized using the PM3 semi-empirical method to a root mean square (RMS) gradient of $0.01 \mathrm{kcal} / \AA$. mol with the Polak-Ribière conjugate gradient algorithm and imported in a mol2 file format. The chrysin structure modeled was 'docked' into the TACE active site (described above) using the FlexX program (27), included in LeadIT Molecular Viewer, version 2.6 (28). FlexX uses a defragmentation procedure on the ligand, places the anchor fragment within the binding site and then incrementally builds the entire ligand. A total of 377 possible docking solutions were generated and ranked using a scoring function that estimates the free energy of binding of the protein-ligand complexes. Preparation of the receptor, ligand and all the post docking interactions were analyzed using the LeadIT molecular viewer. Docking procedures were validated using a re-docking approach, where the reference ligand was docked into the PDB 2FV5 crystal structure using the same procedure and the docking orientation compared with the crystal orientation to judge the reliability of the docking procedure.

Statistical analysis. Statistical analysis was performed with a one-way analysis of the variance procedure using Stata software (version 13; StataCorp LP, College Station, TX, USA). $\mathrm{P}<0.05$ was considered to indicate a statistically significant difference.

\section{Results and Discussion}

Serum ALT and AST activity. The results of 7 days of chrysin administration on serum ALT and AST activities in mice from various treatment groups are shown in Fig. 1. Serum AST and ALT activity was significantly increased $(\mathrm{P}<0.001)$ $24 \mathrm{~h}$ following $\mathrm{CCl}_{4}$ treatment $\left(\mathrm{CCl}_{4}\right.$ group) compared with the control group. Pre-treatment with chrysin $\left(\mathrm{CHR}+\mathrm{CCl}_{4}\right)$ 

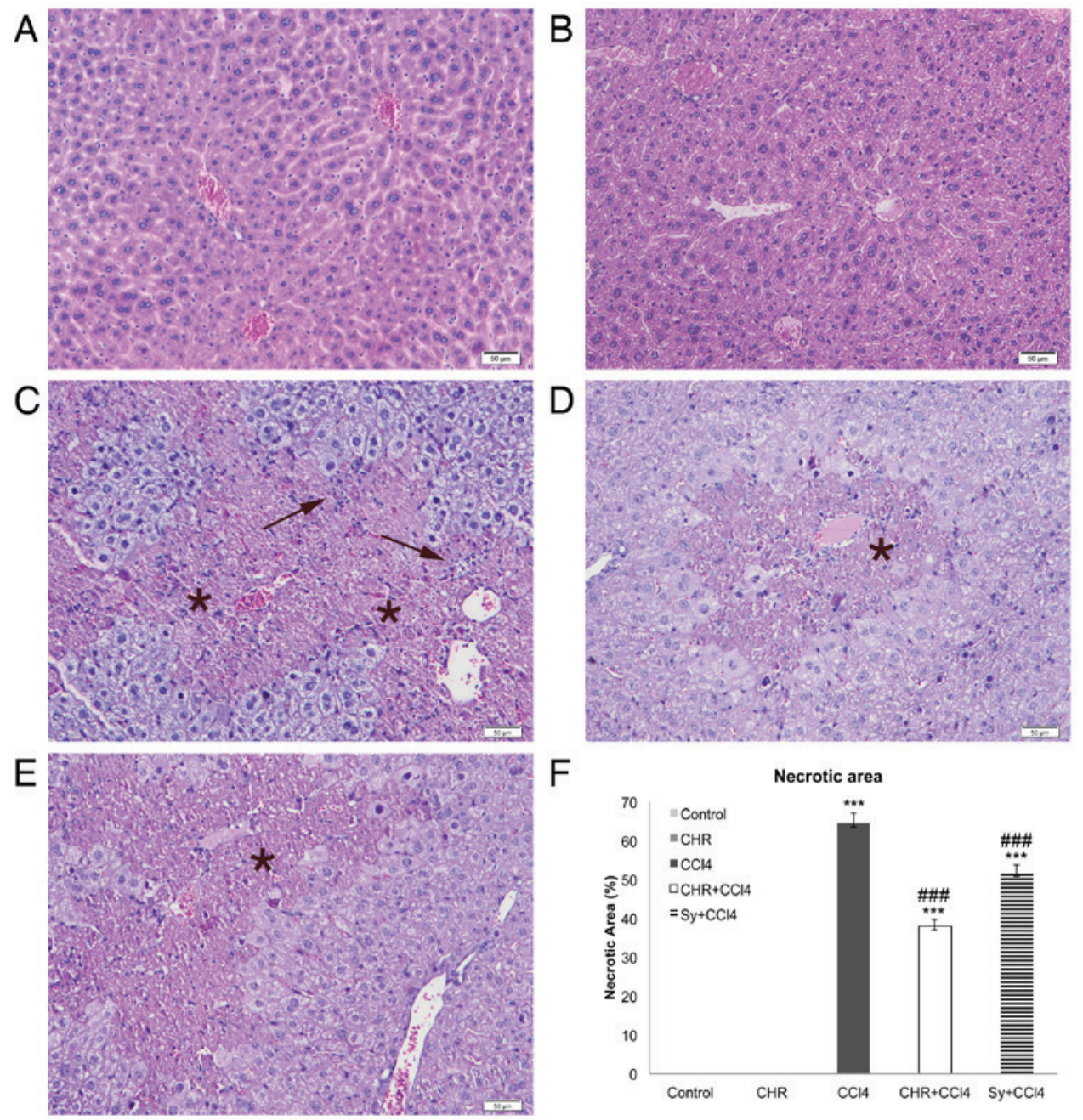

Figure 2. Effect of chrysin on histological changes in the liver of $\mathrm{CCl}_{4}$-treated mice. Representative images of hematoxylin and eosin stained liver sections of the (A) control group, (B) CHR group, (C) $\mathrm{CCl}_{4}$ group, (D) $\mathrm{CHR}+\mathrm{CCl}_{4}$ group and (E) $\mathrm{Sy}+\mathrm{CCl}_{4}$ group. Scale bar, $50 \mu \mathrm{m}$. Necrosis areas are indicated with asterisks, lymphocyte infiltration are indicated by arrows. (F) The percentage of necrotic area in mice liver samples. Results are represented as the mean \pm standard deviation ( $\mathrm{n}=5$ ). Scale bar, $50 \mu \mathrm{m}$. ${ }^{* * *} \mathrm{P}<0.001$ vs. the control group; ${ }^{\# \# *} \mathrm{P}<0.001$ vs. the $\mathrm{CCl}_{4}$ group. $\mathrm{CCl}_{4}$, carbon tetrachloride; CHR, chrysin; Sy, silymarin.

significantly decreased serum aminotransferase activity compared with the $\mathrm{CCl}_{4}$ group $(\mathrm{P}<0.001)$. This result was similar to that of the silymarin pre-treated group $\left(\mathrm{Sy}+\mathrm{CCl}_{4}\right.$; $\mathrm{P}<0.001$ compared with the $\mathrm{CCl}_{4}$ group). The group treated with chrysin and not $\mathrm{CCl}_{4}$ showed no increase in ALT and AST activity compared with the control group.

It is well known that the hepatotoxic agent $\mathrm{CCl}_{4}$ is metabolized to a trichloromethyl radical $(\mathrm{CCl} 3 \bullet)$, which interacts with oxygen to form more free radicals, which damage the liver. Increased membrane permeability from hepatocyte injury leads to leakage of liver enzymes, which causes increased activity of the serum transaminases $(2,29)$. The present study found a significant increase in serum AST and ALT serum activity in mice following $\mathrm{CCl}_{4}$ administration ( $\left.\mathrm{P}<0.001\right)$. Chrysin $(50 \mathrm{mg} / \mathrm{kg})$ pre-treatment significantly decreased serum AST and ALT activity in $\mathrm{CCl}_{4}$-treated mice $(\mathrm{P}<0.001)$, which indicates that chrysin preserves the structural integrity of membranes. This is consistent with the finding of a previous report (16).

Histopathology. The effect of chrysin on histological changes and lipid accumulationin the liver was investigated(Figs.2 and3). Evaluation of liver tissues by light microscopy identified that, compared with the normal liver architecture of the control group (Figs. 2A and 3A), livers of the $\mathrm{CCl}_{4}$ group showed necrotic changes to hepatocytes, which were particularly pronounced in the centrilobular area (Fig. 2C). In addition, inflammatory cell infiltration (Fig. 2C) and microvesicular steatosis of the hepatocytes (Fig. 3C) were detected in the $\mathrm{CCl}_{4}$ group. The group pre-treated with chrysin prior to $\mathrm{CCl}_{4}$ injection (CHR+CCl${ }_{4}$; Figs. 2D and 3D), showed a significant reduction in hepatocellular necrosis and steatosis compared with the $\mathrm{CCl}_{4}$ group $(\mathrm{P}<0.001$; Figs. $2 \mathrm{~F}$ and $3 \mathrm{~F})$. This reduction was greater than that seen in the silymarin group (Figs. 2E and $\mathrm{F}$, and $3 \mathrm{E}$ and $\mathrm{F}$ ). The liver morphology of the group treated with chrysin alone (Figs. 2B and 3B) was comparable with that of the control (Figs. 2A and 3A).

Increased deposition of neutral lipids in hepatocytes is caused by an imbalance between the gain and loss of fatty acids, and the synthesis and excretion of triglycerides (30). This imbalance can be induced by exposure to $\mathrm{CCl}_{4}$, causing micro- and macro-vesicular steatosis (30). The reduction in liver triglyceride accumulation seen in the group pre-treated with chrysin suggests that this flavonoid is able to restore the lipid balance in hepatocytes and thus hepatic function. 

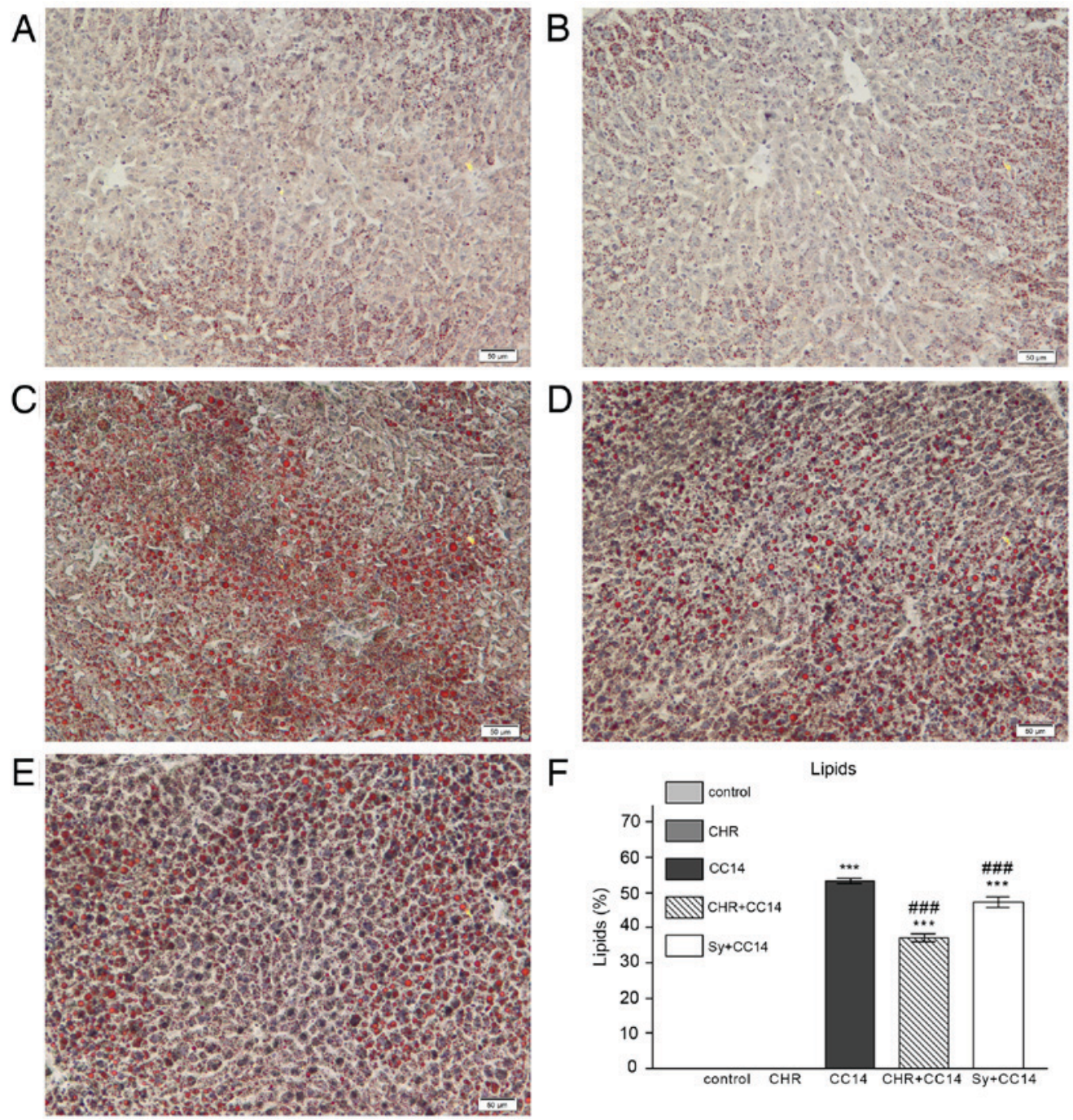

$\mathrm{F}$

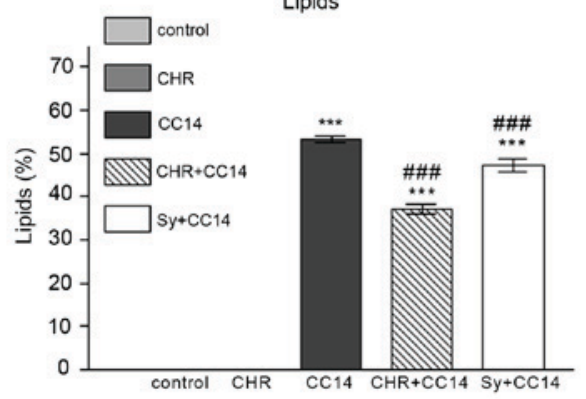

Figure 3. Effect of chrysin on lipid accumulation in the liver of $\mathrm{CCl}_{4}$-treated mice. Representative images of Oil Red $\mathrm{O}$ stained liver sections of the (A) control group, (B) CHR group, (C) $\mathrm{CCl}_{4}$ group, (D) $\mathrm{CHR}+\mathrm{CCl}_{4}$ group and (E) $\mathrm{Sy}+\mathrm{CCl}_{4}$ group. Lipid drops, indicated in red. Scale bar, $50 \mu \mathrm{m}$. (F) The percentage of lipids in mice liver samples. Results are represented as the mean \pm standard deviation $(\mathrm{n}=5)$. Scale bar, $50 \mu \mathrm{m}{ }^{* * *} \mathrm{P}<0.001 \mathrm{vs}$. control; ${ }^{\# \# \# ~} \mathrm{P}<0.001 \mathrm{vs}$. the $\mathrm{CCl} l_{4}$ group. $\mathrm{CCl}_{4}$, carbon tetrachloride; CHR, chrysin; Sy, silymarin.

Liver expression of TNF- $\alpha$ and $\alpha-S M A$ proteins. The effect of chrysin on liver TNF- $\alpha$ and $\alpha$-SMA expression, which is elevated by $\mathrm{CCl}_{4}$, a proinflammatory and profibrotic agent, is shown in Figs. 4 and 5. Significantly increased TNF- $\alpha$ (Fig. 4C) and $\alpha$-SMA (Fig. 5C) expression was observed in the $\mathrm{CCl}_{4}$ group (both $\mathrm{P}<0.001$ compared with the control), particularly in the areas surrounding the centrilobular veins, forming bridges between neighboring veins. TNF- $\alpha$ and $\alpha$-SMA expression was significantly decreased in the livers of chrysin pre-treated mice (Figs. 4D and Fig. 5D) compared with the $\mathrm{CCl}_{4}$ group $(\mathrm{P}<0.001$; Figs. $4 \mathrm{~F}$ and $5 \mathrm{~F})$. This decrease was greater than that seen with silymarin pre-treatment (Figs. 4E and $4 \mathrm{~F}$, and $5 \mathrm{E}$ and $\mathrm{F}$ ). No expression of TNF- $\alpha$ or $\alpha$-SMA was detected in the group treated with chrysin alone (Figs. 4B and 5B) or the control group (Figs. 4A and 5A).

In the present study, chrysin pre-treatment reduced $\mathrm{CCl}_{4}$-induced TNF- $\alpha$ expression. TNF- $\alpha$ is a pro-inflammatory cytokine produced by Kupfer cells, which has been found to be elevated in acute liver diseases and following exposure to hepatotoxic chemicals, including $\mathrm{CCl}_{4}(31,32)$. In this study, the reduction in TNF- $\alpha$ expression caused by chrysin suggests that it serves an important role in attenuating the $\mathrm{CCl}_{4}$-induced inflammatory cascade in the liver. In agreement with the findings of the current study, Ai et al (33) determined potential inhibition of the pro-inflammatory TNF- $\alpha$ pathway by other flavonoids.

Strong hepatic inflammatory responses are accompanied by the necrosis of large areas and the formation of bridges between centrilobular veins (34), causing extended damage to the liver parenchyma. In the present study, these changes were observed in the in the $\mathrm{CCl}_{4}$ group and were reduced by chrysin pre-treatment.

Chronic or severe inflammation can stimulate a fibrotic response, characterized by an irreversible decline in liver function (35). In the present study, chrysin pre-treatment reduced the activation of HSCs, as determined by $\alpha$-SMA expression and the progression of acute hepatic damage into liver fibrosis. $\alpha$-SMA is considered an important factor in the development of liver fibrosis and is thus a marker for HSC (the primary producers of $\alpha$-SMA) activation and fibrous tissue deposition. Therefore, the expression of $\alpha$-SMA is a useful marker for monitoring the efficacy of hepatoprotective therapy. The 

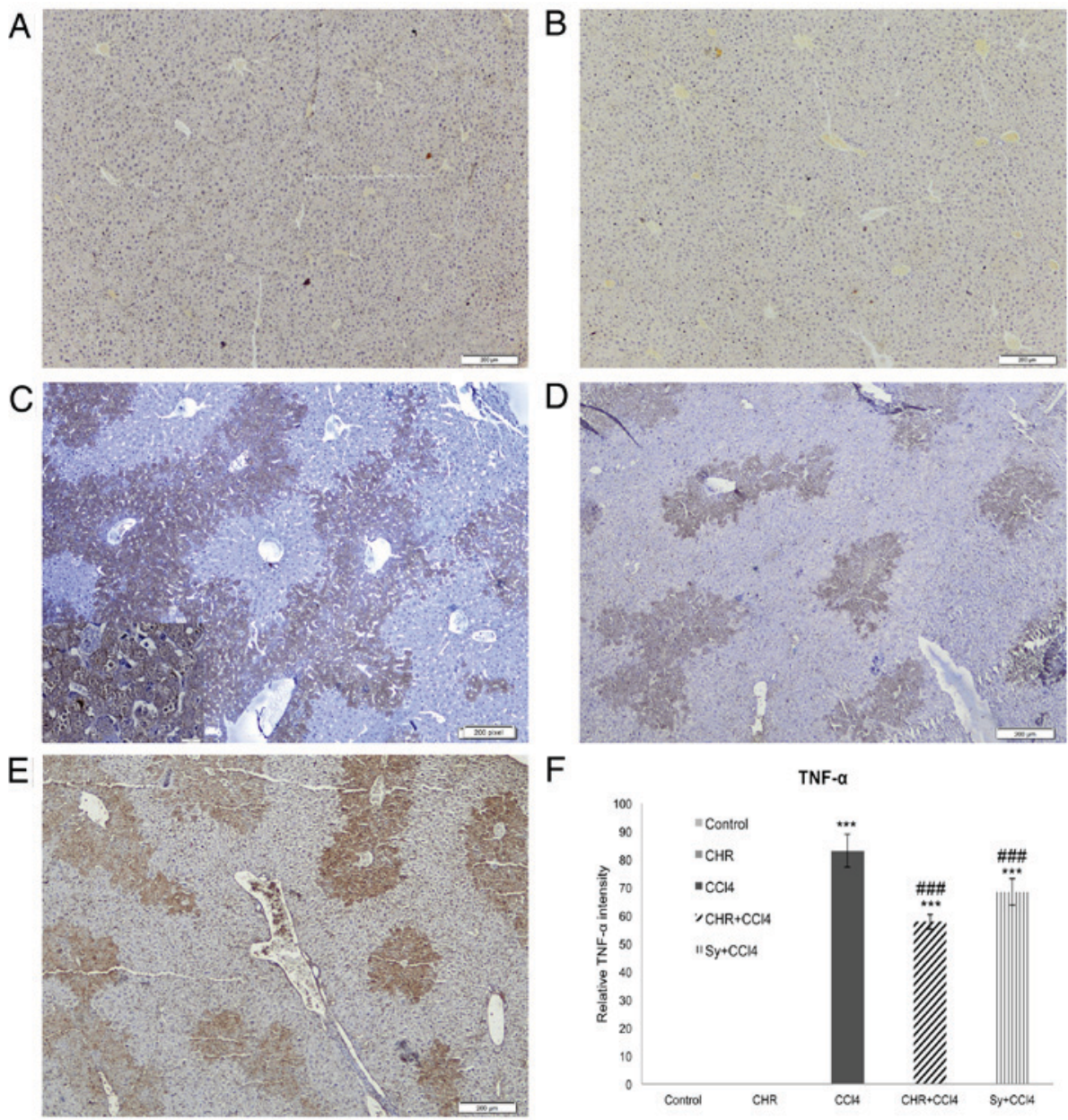

$\mathrm{F}$

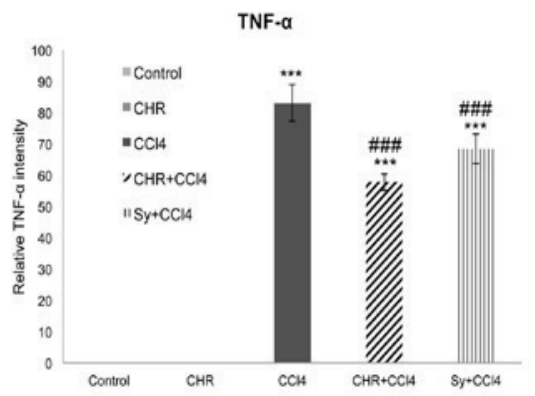

Figure 4. Effect of chrysin on the expression and distribution of TNF- $\alpha$ protein in the liver of $\mathrm{CCl}_{4}$-treated mice. (A) Control group, (B) $\mathrm{CHR}$ group, (C) CCl group, (D) $\mathrm{CHR}+\mathrm{CCl}_{4}$ group and (E) $\mathrm{Sy}+\mathrm{CCl}_{4}$ group. Scale bar, $200 \mu \mathrm{m}$. (F) Quantification of TNF- $\alpha$ staining intensity relative to control and chrysin groups Results are represented as the mean \pm standard deviation $\left(\mathrm{n}=5\right.$ mice per group). ${ }^{* * * *} \mathrm{P}<0.001$ vs. the control group; ${ }^{\# \# \#} \mathrm{P}<0.001$ vs. the $\mathrm{CCl} \mathrm{l}_{4}$ group. TNF- $\alpha$, tumor necrosis factor- $\alpha ; \mathrm{CCl}_{4}$, carbon tetrachloride; CHR, chrysin; Sy, silymarin.

results of the present study identified that $\alpha$-SMA expression in liver tissue from the $\mathrm{CCl}_{4}$ group was significantly increased compared with the control group $(\mathrm{P}<0.001)$. A previous study reported that a reduction in $\alpha$-SMA expression was accompanied by a decrease in the quantity of activated HSCs (36). The results of the present study showed that $\alpha$-SMA expression in $\mathrm{CCl}_{4}$-injured livers was reduced by chrysin, which indicates that chrysin deactivates HSCs.

Electron microscopy. Electron microscopy revealed a normal hepatocyte ultrastructure in the control group (Fig. 6A) and chrysin alone (Fig. 6B), with regularly shaped nuclei and rough endoplasmic reticuli (rER), and few lipid globules. However, hepatocytes of the $\mathrm{CCl}_{4}$ group showed lipid globule accumulation, organelle degeneration and proliferation of smooth ER (sER) vesicles (Fig. 6C). Pre-treatment with chrysin markedly reduced lipid globule enlargement and quantity (Fig. 6D), similarly to the silymarin pre-treated group (Fig. 6E).

The results of the present study identified that organelle and cytoplasmic structures were protected against hepatotoxic effects of $\mathrm{CCl}_{4}$ by chrysin pre-treatment including membranes preservation. A previous study demonstrated that membrane damage causes alterations in lipoprotein and lipid droplet accumulation in hepatocytes (37). Lipid accumulation is accompanied by dilatations and focal breaks of rER cisternae, likely due to membrane structure damage caused by lipid peroxidation (38). In addition, a previous study showed that the flavonoids provided protection against free radicals generated by xenobiotic biotransformation and lipid peroxidation (16).

Numerous xenobiotics are metabolized in oxidase chain reactions, including that of the cytochrome P450 system. Located primarily in centrlobular hepatocytes, cytochrome P450 enzymes are associated with drug-induced sER proliferation (39). In the present study, sER proliferation was evident in electron microscopy micrographs of the $\mathrm{CCl}_{4}$ treated group. Furthermore, chrysin reduced $\mathrm{CCl}_{4}$-induced effects on hepatocyte ultrastructure, similarly to silymarin at the same dose.

Molecular modeling. The present study demonstrated that the hepatoprotective activity of chrysin is mediated through TNF- $\alpha$. Chrysin pre-treatment significantly reduced $\mathrm{CCl}_{4}$-induced TNF- $\alpha$ protein expression $(\mathrm{P}<0.001)$. This indicates that chrysin may modulate TNF- $\alpha$ processing, reducing soluble TNF- $\alpha$ generation. TNF- $\alpha$ is synthesized as a membrane-anchored precursor and the soluble form of TNF- $\alpha$ is released into the extracellular space through limited proteolysis by the zinc-endopeptidase TACE (40). TACE is a 

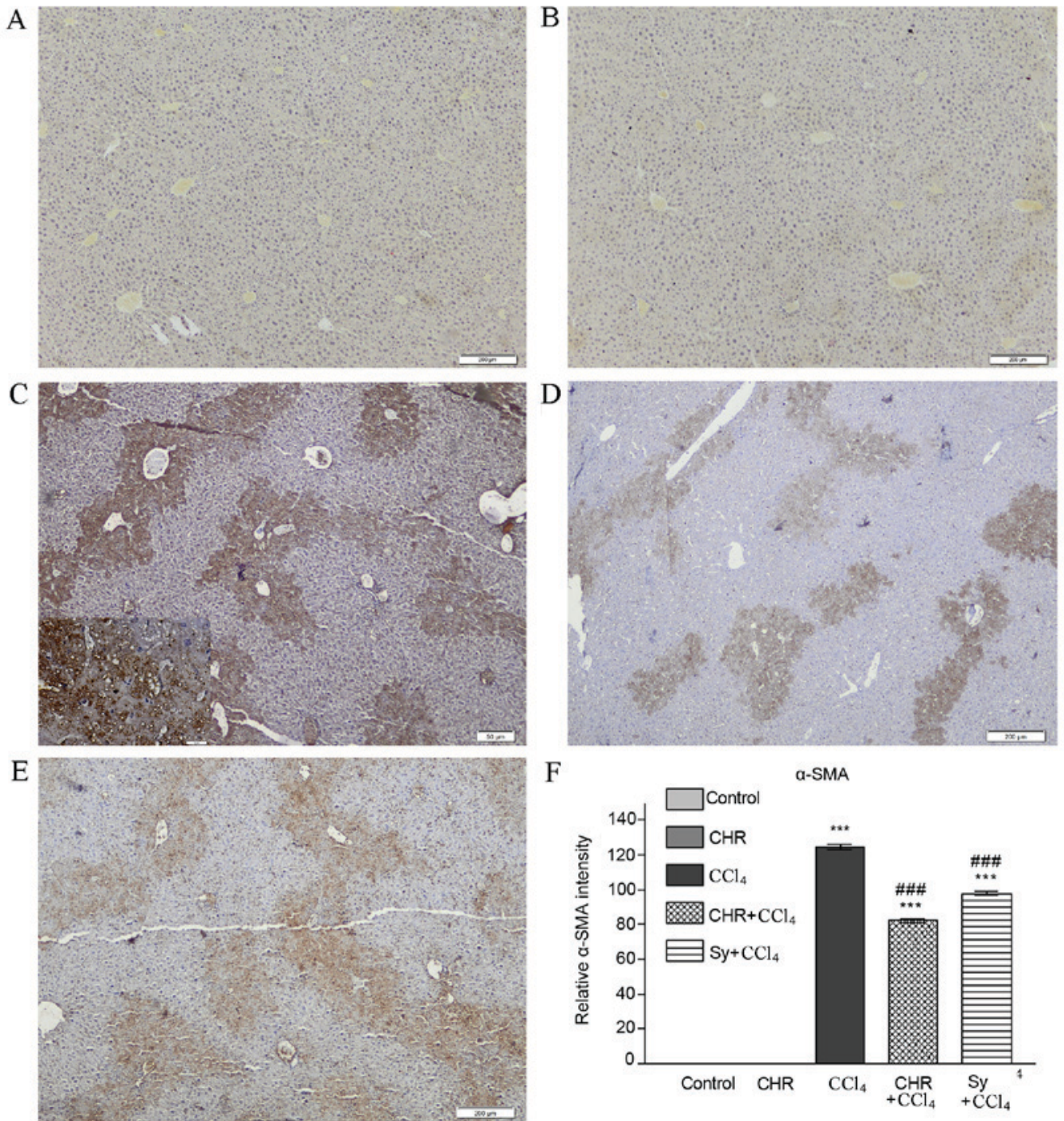

Figure 5. Effect of chrysin on the expression and distribution of $\alpha$-SMA protein in the liver of $\mathrm{CCl}_{4}$-treated mice. (A) Control group, (B) $\mathrm{CHR}$ group, (C) CCl group, (D) $\mathrm{CHR}+\mathrm{CCl}_{4}$ group and (E) $\mathrm{Sy}+\mathrm{CCl}_{4}$ group. Scale bar, $200 \mu \mathrm{m}$. (F) Quantification of $\alpha$-SMA staining intensity. Results are represented as the mean \pm standard deviation $(\mathrm{n}=5)$. ${ }^{* * *} \mathrm{P}<0.001$ vs. the control group; ${ }^{\# \# *} \mathrm{P}<0.001$ vs. the $\mathrm{CCl}_{4}$ group. $\alpha$-SMA, $\alpha$-smooth muscle actin; $\mathrm{CCl}_{4}$, carbon tetrachloride; CHR, chrysin; Sy, silymarin.

multi-domain peptidase consisting of an extracellular region, a transmembrane helix and an intracellular C-terminal tail. The extracellular region of TACE comprises an N-terminal pro-domain, a 259 amino acid residue catalytic domain and a disintegrin-like cysteine-rich domain (40). The catalytic domain of TACE recognizes the pro-TNF- $\alpha$ cleavage site (Ala76-Val77) to generate TNF- $\alpha$.

The present study investigated the theory of chrysin interacting with the TACE active site using the FlexX program. The crystal structure of TACE in complex with the inhibitor IK682 (PDB ID: 2FV5) (25) was used for a docking study (results shown in Fig. 7), which was validated by a re-docking procedure. FlexX reliably reproduced the crystallographic orientation of bound IK682 with an RMSD of $2.2 \AA$ (Fig. 7). Comparison of the pharmacophoric features of the ligand-enzyme interaction in FlexX revealed the most favorable binding energy solution (Fig. 7A), which elucidated the pharmacophoric interaction for TACE inhibition as observed in the crystal structure (Fig. 7B). Therefore, FlexX accurately docked IK682 within the TACE active site (Fig. 7, upper right).
This methodology was then applied to investigate docking of chrysin within the TACE active site.

Molecular docking of chrysin revealed that chrysin favorably binds to the active site of TACE, with an estimated binding energy of $-23.5 \mathrm{~kJ} / \mathrm{mol}$. In addition, chrysin was found to dock close to the catalytic region of TACE. The lowest energy docking solution revealed that the chromone moiety of chrysin, composed of A-, B- and C-rings, was oriented towards the catalytic zinc residue in the hydrophobic S1 sub-pocket of TACE, with the B-ring deep in the hydrophobic S3 sub-pocket (Fig. 8A). This binding of chrysin is non-planar, with the B-ring tilted $\sim 45^{\circ}$ in respect to the chromone plane (Fig. 8A). Fig. 8A shows essential interactions of chrysin with key catalytic residues of TACE. The catalytic zinc residue of TACE is coordinated by three imidazole nitrogen atoms of His-405, His-409 and His-415. The 7-OH group of TACE-docked chrysin was $4.5 \AA$ away from the catalytic zinc and thus able to coordinate with it. This interaction may displace the 'catalytic' water molecule from the active site of the enzyme, explaining the inhibition 

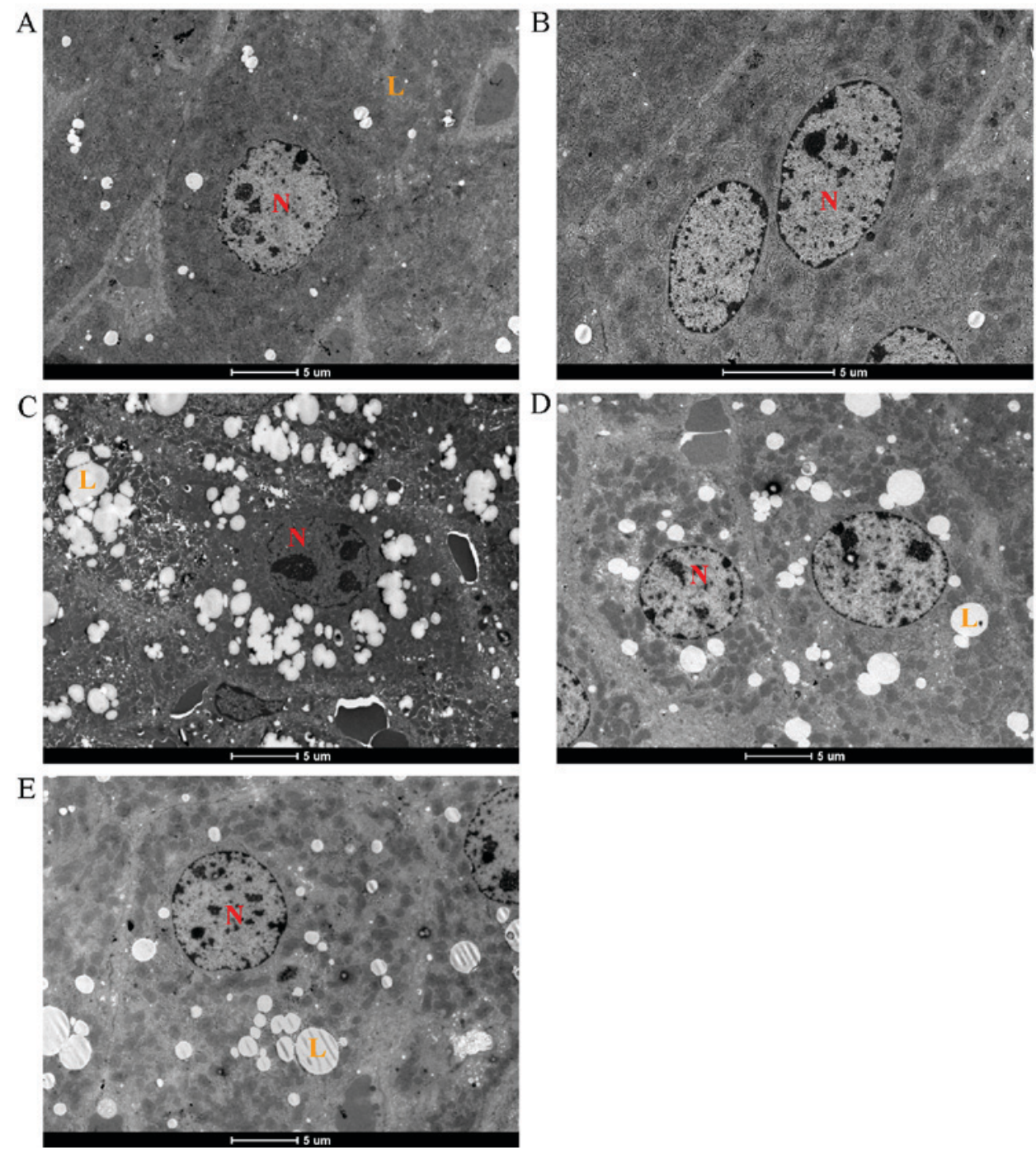

Figure 6. Effect of chrysin on hepatocyte ultrastructure in $\mathrm{CCl}_{4}$-treated mice. (A) The control group showed normal N and L. (B) $\mathrm{CHR}$ group. (C) The CCl group showed an edematous cytoplasm matrix with sER proliferation, and an increased quantity and size of L. (D) The $\mathrm{CHR} \mathrm{CCl}_{4}$ group showed a reduction in the quantity and size of $\mathrm{L}$, and no sER proliferation. (E) The $\mathrm{Sy}+\mathrm{CCl}_{4}$ group showed a reduction in the quantity and size of $\mathrm{L}$, and no sER proliferation. Transmission electron miscopy images representative of the group. Scale bar, $50 \mu \mathrm{m}$. N, nucleus; L, lipid drop; sER, smooth endoplasmic reticulum.

of TACE activity by chrysin. In addition, the 5-OH group of chrysin forms a hydrogen bond with the carboxylate oxygen of Glu-406 in TACE, which acts as a base during catalysis (41). Furthermore, the 7-OH group of chrysin forms a hydrogen bond with Pro-437 of TACE, which serves a key role in reversing the Met-turn produced by Tyr-433, Val-434, Met-435 and Tyr-436 to form the outer wall of the S1 crevice (39). The hydrogen bonding seen positioned the outer wall loop of the S1 sub-pocket in such a way that it essentially blocked the cavity opening. All these interactions stabilize the TACE-chrysin closed complex.

Fig. 8B represents the surface of the chrysin-bound TACE active site, colored according to B-factor value (flexibility). Chrysin was found to bind deep inside the hydrophobic cavity and the flexible Met-turn closes the cavity opening, which is bridged by Ala- 439 and Leu-348. The essential pharmacophoric interaction of TACE inhibition by chrysin is shown in Fig. 8C. Excluding the two hydrogen bonds (described above), the majority of the interactions were hydrophobic. The hydrophobic B-ring of chrysin is oriented inside the $\mathrm{S} 3$ sub-pocket framed by a number of hydrophobic residues, including Leu-401, Val-402, Ile-438, Ala-439 and Val-440.

In conclusion, the results of the present study suggest that inflammation signaling pathways were activated in the pathogenesis of $\mathrm{CCl}_{4}$-induced acute hepatic damage and that this could be counteracted with seven days of chrysin pre-treatment. In addition, the hepatoprotective activity of chrysin was identified to be mediated through TNF- $\alpha$, via chrysin reducing soluble TNF- $\alpha$ generation via inhibiting TACE. Furthermore, chrysin was demonstrated to be a potent hepatoprotective agent, with similar effects to silymarin at the same dose, and should be investigated as a targeted drug to maintain a healthy liver and prevent liver damage.

\section{Acknowledgements}

The authors thank Dr Soumalee Basu, Coordinator of the Centre for High Performance Computing for Modern Biology, Ballygunge Science College, University of Calcutta, Kolkata, West Bengal, India, for access to HYPERCHEM version 8.0 
A

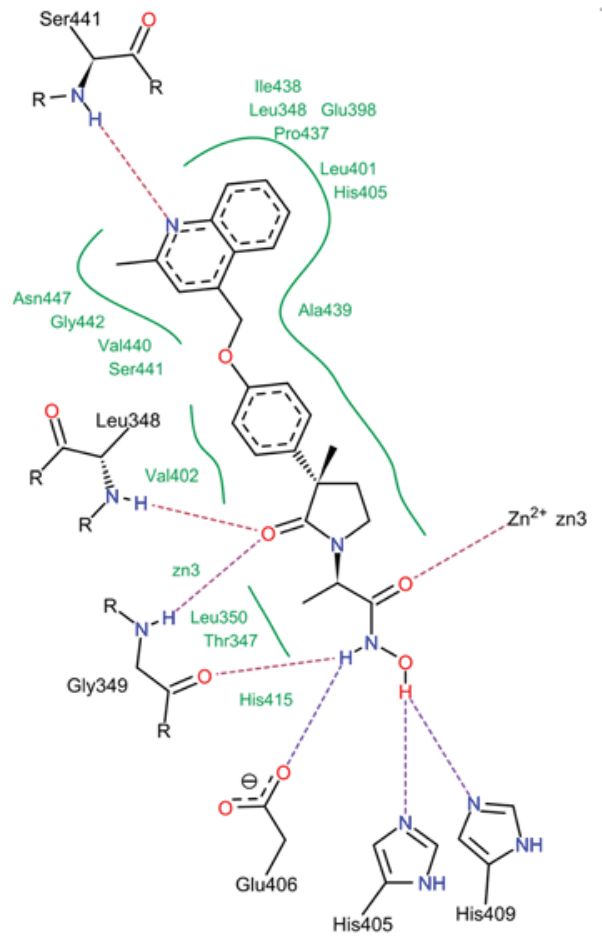

B

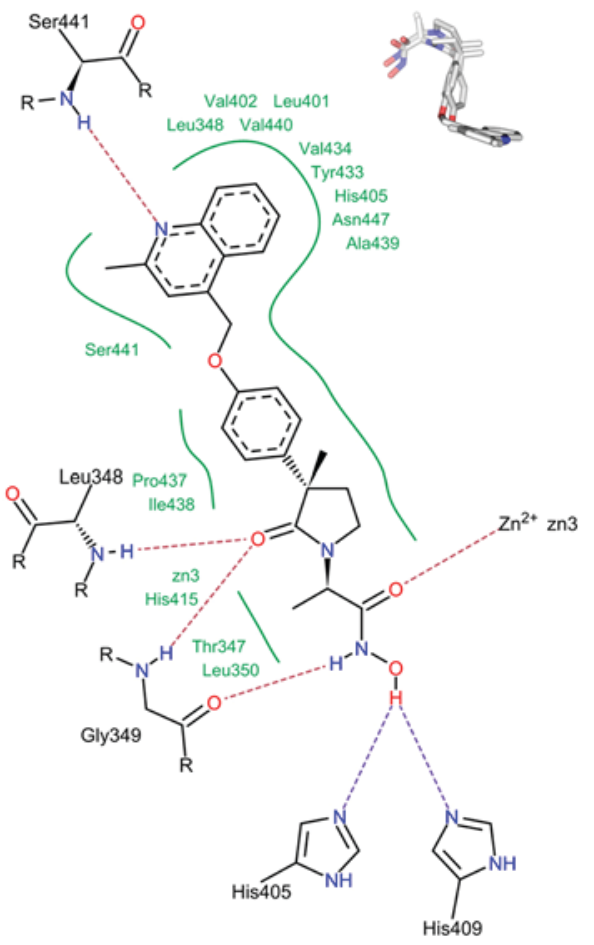

Figure 7. Validation of the FlexX docking method through re-docking of the inhibitor IK682 into the crystal structure (PDB ID: 2FV5) of tumor necrosis factor- $\alpha$ converting enzyme (TACE). (A) Pharmacophoric features of TACE interaction with inhibitor IK682, obtained using the FlexX docking method. (B) Pharmacophoric interaction of TACE with inhibitor IK682, as observed in the crystal structure. Top right, structure of the inhibitor IK682, obtained from docking with the orientation observed in the crystal structure. Amino acid names are abbreviated using standard International Union of Pure and Applied Chemistry convention. Green lines represent hydrophobic interactions, while dashed lines represent hydrogen bonds and metal interactions.

A

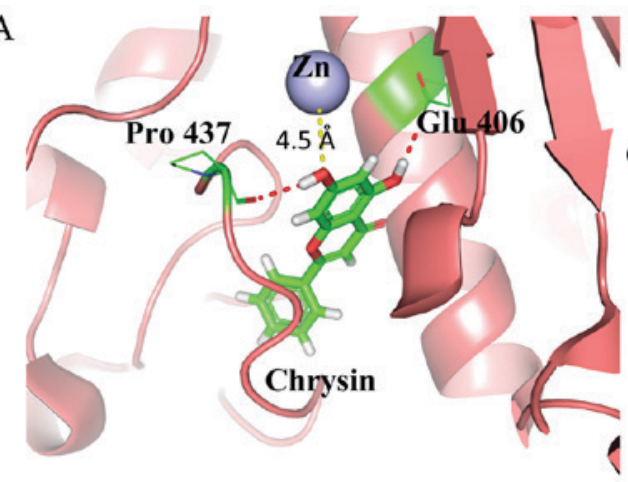

$\mathrm{C}$

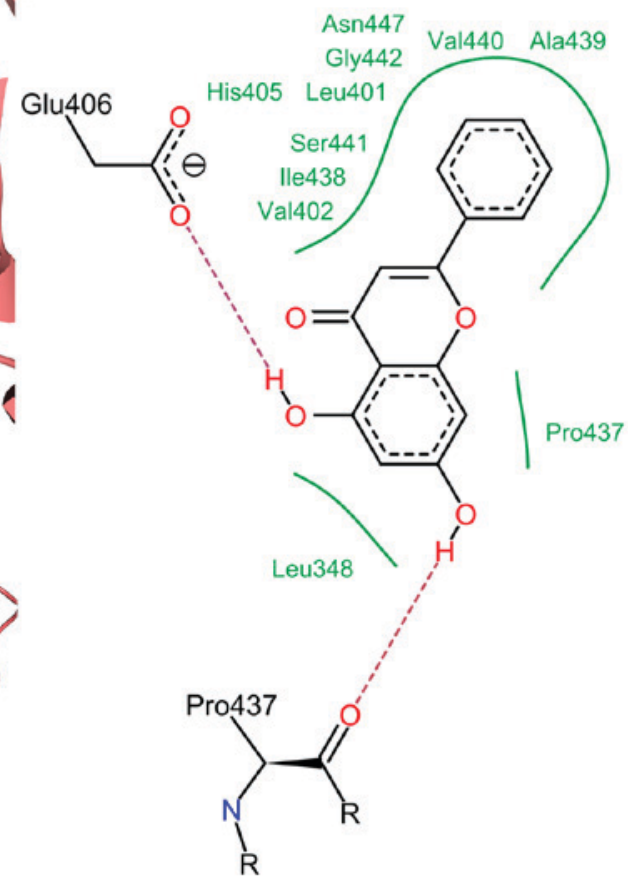

B

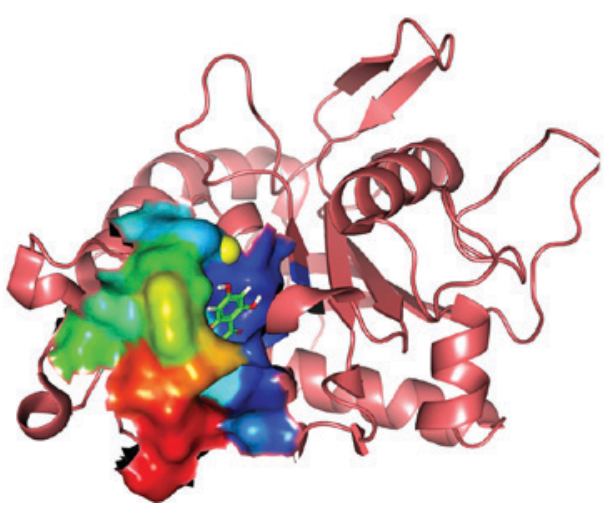

Figure 8. Structure of the lowest energy docking solution of the tumor necrosis factor- $\alpha$ converting enzyme (TACE)-chrysin complex obtained from molecular modeling. (A) Interactions of docked chrysin within the TACE binding site. Chrysin is rendered as a stick representation, TACE is rendered as a ribbon representation and hydrogen bonds indicated by dashed lines. (B) Orientation of docked chrysin within the active site of TACE. The active site is represented in surface mode and colored according to B-factor value (flexibility), with blue to green to red signifying increasing flexibility. (C) The essential pharmacophoric interactions of TACE inhibition by chrysin. Hydrogen bonds are indicated by dashed lines. Amino acid names are abbreviated using standard International Union of Pure and Applied Chemistry convention. Green lines represent hydrophobic interactions, while dashed lines represent hydrogen bonds. 
and the FlexX docking package. The present study was supported by the Fundación Séneca del Centro de Coordinación de la Investigación, Murcia, Spain (grant no 18946/JLI/13) and the NILS Science and Sustainability Individual Mobility of Researchers grant, funded by the European Economic Area financial mechanism (grant no. 012-ABEL-CM-2014A).

\section{References}

1. Kaminski M and Wiaderkiewicz R: The role of the liver in xenobiotic biotransformation. Part I. The role of the liver and its cells and their interactions. Problems of Forensic Sciences LXXII: 357-378, 2007.

2. Manibusan MK, Odin M and Eastmon DA: Postulated carbon tetrachloride mode of action: A review. J Environ Sci Health C Environ Carcinog Ecotoxicol Rev 25: 185-209, 2007.

3. Weber LW, Boll M and Stampfl A: Hepatotoxicity and mechanism of action of haloalkanes: Carbon tetrachloride as a toxicological model. Crit Rev Toxicol 33: 105-136, 2003.

4. Dong D, Zhang S, Yin L, Tang X, Xu Y, Han X, Qi Y and Peng J: Protective effects of the total saponins from Rosa laevigata Michx fruit against carbon tetrachloride-induced acute liver injury in mice. Food Chem Toxicol 62: 120-130, 2013.

5. Kamel R and El Morsy EM: Hepatoprotective effect of methylsulfonylmethane against carbon tetrachloride-induced acute liver injury in rats. Arch Pharm Res 36: 1140-1148, 2013.

6. Pfeffer K: Biological functions of tumor necrosis factor cytokines and their receptors. Cytokine Growth Factor Rev 14: 185-191, 2003.

7. Alric L, Pinelli E, Carrera G, Vinel JP, Beraud M, Duffaut M, Pascal JP and Pipy B: Involvement of calcium in macrophage leukotriene release during experimental cirrhosis. Hepatology 23 614-622, 1996.

8. Tarras N, Moles A, Morales A, García-Ruiz C, FernándezCheca JC and Marí M: Critical role of tumor necrosis factor receptor 1 , but not 2 , in hepatic stellate cell proliferation, extracellular matrix remodeling and liver fibrogenesis. Hepatology 54 319-327, 2011

9. Friedman SL: Molecular regulation of hepatic fibrosis, an integrated cellular response to tissue injury. J Biol Chem 275 2247-2250, 2000.

10. Leask A and Abraham D: TGF-beta signaling and the fibrotic response. FASEB J 18: 816-827, 2004.

11. Lobo V, Patil A, Phatak A and Chandra N: Free radicals, antioxidants and functional foods: Impact on human health. Pharmacogn Rev 4: 118-126, 2014.

12. Pietta PG: Flavonoids as Antioxidants. J Nat Prod 63: 1035-1042, 2000.

13. Abascal K and Yarnell E: The many faces of Silybum marianum (Milk Thistle): Part 2- clinical uses, safety and types of preparations. Alternative and Complementary Therapies 9: 251-256, 2003.

14. Kshirsagar A, Ingawale D, Ashok P and Vyawahare N: Silymarin: A comprehensive review. Phog Rev 3: 126-134, 2009.

15. Siess MH, Le Bon AM, Canivenc-Lavier MC, Amiot MJ, Sabatier S, Aubert SY and Suschetet M: Flavonoids of honey and propolis: Characterization and effects on hepatic drug metabolizing enzymes and benzo[a]pyrene-DNA binding in rats. J Agric Food Chem 44: 2297-2301, 1996.

16. Anand KV, Anandhi R, Pakkiyaraj M and Geraldine P: Protective effect of chrysin on carbon tetrachloride (CCl4)-induced tissue injury in male Wistar rats. Toxicol Ind Health 27: 923-933, 2011.

17. Pushpavalli G, Kalaiarasi P, Veeramani C and Pugalendi KV: Effect of chrysin on hepatoprotective and antioxidant status in D-galactosamine-induced hepatitis in rats. Eur J Pharmacol 631: 36-41, 2010.

18. Brechbuhl HM, Kachadourian R, Min E, Chan D and Day BJ: Chrysin enhances doxorubicin-induced cytotoxicity in human lung epithelial cancer cell lines: The role of glutathione. Toxicol Appl Pharmacol 258: 1-9, 2012.

19. Bae Y, Lee S and Kim SH: Chrysin suppresses mast cell-mediated allergic inflammation: Involvement of calcium, caspase-1 and nuclear factor-кB. Toxicol Appl Pharmacol 254: 56-64, 2011.
20. Shin EK, Kwon HS, Kim YH, Shin HK and Kim JK: Chrysin, a natural flavone, improves murine inflammatory bowel diseases. Biochem Biophys Res Commun 381: 502-507, 2009.

21. Balta C, Herman H, Boldura OM, Gasca I, Rosu M, Ardelean A and Hermenean A: Chrysin attenuates liver fibrosis and hepatic stellate cell activation through TGF-b/Smad signaling pathway. Chem Biol Interact 240, 94-101, 2015.

22. Khan MS, Devaraj H and Devaraj N: Chrysin abrogates early hepatocarcinogenesis and induces apoptosis in N-nitrosodiethylamine-induced preneoplastic nodules in rats. Toxicol Appl Pharmacol 251: 85-94, 2011.

23. Phan TA, Yu XM, Kunnimalaiyaan M and Chen H: Antiproliferative effect of chrysin on anaplastic thyroid cancer. J Surg Res 170: 84-88, 2011.

24. Khan R, Khan AQ, Qamar W, Lateef A, Tahir M, Rehman MU, Ali F and Sultana S: Chrysin protects against cisplatin-induced colon. toxicity via amelioration of oxidative stress and apoptosis: Probable role of p38MAPK and p53. Toxicol Appl Pharmacol 258: 315-329, 2012.

25. Niu X, Umland S, Ingram R, Beyer BM, Liu YH, Sun J, Lundell D and Orth P: IK682, a tight binding inhibitor of TACE. Arch Biochem Biophys 451: 43-50, 2006.

26. Hyperchem, Hypercube, Inc., USA, 2002.

27. Rarey M, Kramer B, Lengauer T and Klebe G: A fast flexible docking method using an incremental construction algorithm. J Mol Biol 261: 470-489, 1996.

28. Patel DS and Bharatam PV: New leads for selective GSK-3 inhibition: Pharmacophore mapping and virtual screening studies. J Comput Aided Mol Des 20: 55-66, 2006.

29. Clawson GA: Mechanisms of carbon tetrachloride hepatotoxicity. Pathol Immunopathol Res 8: 104-112, 1989.

30. Wahlang B, Beier J, Clair H, Bellis-Jones HJ, Falkner KC, McClain CJ and Cave MC: Toxicant-associated steatohepatitis. Toxicol Pathol 41: 343-360, 2013.

31. Brenner C, Galluzzi L, Kepp O and Kroemer G: Decoding cell death signals in liver inflammation. J Hepatol 59: 583-594, 2013.

32. Orfila C, Lepert JC, Alric L, Carrera G, Beraud M, Vinel JP and Pipy B: Expression of TNF-alpha and immunohistochemical distribution of hepatic macrophage surface markers in carbon tetrachloride-induced chronic liver injury in rats. Histochem J 31: 677-685, 1999.

33. Ai G, Liu Q, Hua W, Huang Z and Wang D: Hepatoprotective evaluation of the total flavonoids extracted from flowers of Abelmoschus manihot (L.) Medic: In vitro and in vivo studies. J Ethnopharmacol 146: 794-802, 2013.

34. Domitrović R, Jakovac H and Blagojević G: Hepatoprotective activity of berberine is mediated by inhibition of TNF- $\alpha$, $\mathrm{COX}-2$, and iNOS expression in $\mathrm{CCl}(4)$-intoxicated mice. Toxicology 280: 33-43, 2011.

35. Friedman SL: Mechanisms of hepatic fibrogenesis. Gastroenterology 134: 1655-1669, 2008.

36. Kim M, Yang SG, Kim JM, Lee JW, Kim YS and Lee JI: Silymarin suppresses hepatic stellate cell activation in a dietary rat model of non-alcoholic steatohepatitis: Analysis of isolated hepatic stellate cells. Int J Mol Med 30: 473-479, 2012.

37. Ozturk F, Gul M, Ates B, Ozturk IC, Cetin A, Vardi N, Otlu A and Yilmaz I: Protective effect of apricot (Prunus armeniaca L.) on hepatic steatosis and damage induced by carbon tetrachloride in Wistar rats. Br J Nutr 102: 1767-1775, 2009.

38. Tasci I, Mas N, Mas MR, Tuncer M and Comert B: Ultrastructural changes in hepatocytes after taurine treatment in $\mathrm{CCl}_{4}$ induced liver injury. World J Gastroenterol 14: 4897-4902, 2008.

39. Cheville N: Ultrastructural pathology and interorgannelle cross talk in hepatotoxicity. Toxicol Pathol 41: 210-226, 2013.

40. Maskos K, Fernandez-Catalan C, Huber R, Bourenkov GP, Bartunik H, Ellestad GA, Reddy P, Wolfson MF, Rauch CT, Castner BJ, et al: Crystal structure of the catalytic domain of human tumor necrosis factor-alpha-converting enzyme. Proc Natl Acad Sci USA 95: 3408-3412, 1998.

41. Grams F, Reinemer P, Powers JC, Kleine T, Pieper M, Tschesche H, Huber R and Bode W: X-ray structures of human neutrophil collagenase complexed with peptide hydroxamate and peptide thiol inhibitors. Implications for substrate binding and rational drug design. Eur J Biochem 228: 830-841, 1995. 